\title{
THE INEQUALITIES OF RANDOMLY WEIGHTED SUMS OF PAIRWISE NQD SEQUENCES AND ITS APPLICATION TO LIMIT THEORY
}

\author{
Xiaoqin Li, Zhangrui Zhao, WenZhi Yang And Shuhe Hu
}

Abstract. By using some inequalities of randomly weighted sums of pairwise NQD random variables, we investigate the single-indexed randomly weighted and double-indexed randomly weighted sums of these dependence structure. Some almost sure convergence and complete convergence results are obtained, which extend the corresponding results for the nonweighted and constant weighted cases to the case of randomly weighted. Last, some simulations are also illustrated in this paper.

Mathematics subject classification (2010): 60F15.

Keywords and phrases: Pairwise NQD sequences, randomly weighted, complete convergence.

\section{REFERENCES}

[1] A. AdLER, A. RosALS Ky, Some general strong laws for weighted sums of stochastically dominated random variables, Stoch. Anal. Appl. 5 (1987), 1-16.

[2] A. Adler, A. ROSAlSky, R. L. TAYLOR, Strong laws of large numbers for weighted sums of random elements in normed linear spaces, Int. J. Math. Math. Sci. 12 (1989), 507-530.

[3] A. V. Bulinski, A. Shas Kin, Limit Theorems for Associated Random Fields and Related Systems, World Scientific, Singapore, 2007.

[4] P. Y. Chen, S. H. Sung, Generalized Marcinkiewicz-Zygmund type inequalities for random variables and applications, J. Math. Inequal. 10 (2016), 837-848.

[5] Z. Y. Chen, T. T. LiU, J. M. LinG, X. J. WANG, On the convergence rate for arrays of row-wise NOD random variables, Comm. Statist. Theory Methods 45 (2016), 1215-1223.

[6] X. DenG, X. J. WANG, Y. Wu, Y. Ding, Complete moment convergence and complete convergence for weighted sums of NSD random variables, RACSAM, 110 (2016), 97-120.

[7] S. X. GAN, P. Y. CHEN, Some limit theorems for sequences of pairwise NQD random variables, Acta Math. Sci. Ser. B Engl. Ed. 28 (2008), 269-281.

[8] S. Ghosal, T. K. Chandra, Complete convergence of martingale arrays, J. Theor. Probab. 11 (1998), 621-631.

[9] D. L. Hanson, F. T. Wright, A bound on tail probabilities for quadratic forms in independent random variables, Ann. Math. Statist. 42 (1971), 1079-1083.

[10] S. H. Hu, X. T. Liu, X. H. WAnG, X. Q. LI, Strong law of large numbers of partial sums for pairwise NQD sequences, J. Math. Res. Appl. 33 (2013), 111-116.

[11] H. JABBARI, On almost sure convergence for weighted sums of pairwise negatively quadrant dependent random variables, Statist. Papers 54 (2013), 765-772.

[12] K. JoAG-Dev, F. Proschan, Negative association of random variables with applications, Ann. Statist. 11 (1983), 286-295.

[13] E. L. Lehmann, Some concepts of dependence, Ann. Math. Statist. 37 (1966), 1137-1153.

[14] L. LiU, Precise large deviations for dependent random variables with heavy tails, Stat. Probab. Lett. 79 (2009), 1290-1298.

[15] S. H. SUNG, Strong limit theorems for pairwise NQD random variables, Comm. Statist. Theory Methods 42 (2013), 3965-3973.

[16] S. H. SUNG, Convergence in $r$-mean of weighted sums of NQD random variables, Appl. Math. Lett. 26 (2013), 18-24. 
[17] X. J. WANG, S. H. HU, The consistency of the nearest neighbor estimator of the density function based on WOD samples, J. Math. Anal. Appl. 429 (2015), 497-512.

[18] X. J. Wang, T.-C. Hu, A. Volodin, S. H. HU, Complete convergence for weighted sums and arrays of rowwise extended negatively dependent random variables, Comm. Statist. Theory Methods 42 (2013), 2391-2401.

[19] X. J. WANG, Y. WU, S. H. HU, Exponential probability inequality for m-END random variables and its applications, Metrika, 79 (2016), 127-147.

[20] F. T. WRIGHT, A bound on tail probabilities for quadratic forms in independent random variables whose distributions are not necessarily symmetric, Ann. Probab. 1 (1973), 1068-1070.

[21] Q. Y. WU, Convergence properties of pairwise NQD random sequences, Acta Math. Sin. Chin. Ser. 45 (2002), 617-624.

[22] Q. Y. WU, Complete convergence for negatively dependent sequences of random variables, J. Inequal. Appl. 2010 (2010), Article ID 507293, 10 pages.

[23] Q. Y. WU, Y. Y. JIANG, The strong law of large numbers for pairwise NQD random variables, J. Syst. Sci. Complex. 24 (2011), 347-357.

[24] Y. F. WU, Some limit theorems for arrays of rowwsie pairwise negatively quadratic dependent random variables, Theory Probab. Appl. 59 (2015), 344-354.

[25] Y. F. WU, R. ANDREW, Strong convergence for m-pairwise negatively quadrant dependent random variables, Glas. Mat. Ser. III 50 (2015), 245-259.

[26] Y. F. WU, M. L. GUO, A note on strong law of large numbers for partial sums of pairwise NQD random variables, J. Math. Res. Appl. 34 (2014), 231-239.

[27] Y. F. WU, G. J. SHEN, On convergence for sequences of pairwise negatively quadrant dependent random variables, Appl. Math. 59 (2014), 473-487.

[28] W. Z. YANG, S. H. HU, Complete moment convergence of pairwise NQD random variables, Stoch.: Int. J. Probab. Stoch. Process. 87 (2015), 199-208. 